\title{
Neuro-Behçet disease presented with pachymeningitis in a child
}

\author{
Gülsüm Alkan, M.D. ${ }^{a}$, Ayşe Kartal, M.D. Associate Professor ${ }^{b}$, Melike Emiroğlu, M.D. Associate Professorc and \\ Yahya Paksoy M.D. Professor ${ }^{d}$
}

\begin{abstract}
Behçet's disease (BD) is a rare systemic vasculitis disorder of unknown etiology characterized by recurrent oral and genital apthae and uveitis. It involves the central or peripheral nervous system; occurs rarely during childhood. Isolated acute aseptic meningitis is extremely uncommon. We report here a case of Neuro-Behçet disease(NBD) diagnosed in a 14-year-old girl. The patient presented acute headache, diplopia, papilla edema, and meningeal irritation. She had a history of recurrent oral ulcers. Brain magnetic resonance imaging revealed pachymeningitis. Pleocytosis and pressure increase were the cerebrospinal fluid findings. Although medical therapy, her complaints were not resolved. Uveitis was not detected, pathergy test was negative. HLA-B51 allele was positive. The findings were considered to unusual NBD. The patient improved dramatically after steroid therapy. BD should be considered in differential diagnosis of meningitis unless an infectious agent is demonstrated. To our knowledge, a case of pachymeningitis with NBD, was not described in children.

Key words: Behçet's syndrome, aseptic meningitis, children.
\end{abstract}

http:/ / dx.doi.org/10.5546/ aap.2019.eng.e644

To cite: Alkan G, Kartal A, Emiroglu M, Paksoy Y. NeuroBehçet disease presented with pachymeningitis in a child. Arch Argent Pediatr 2019;117(6):e644-e647. a Malatya Training and Research Hospital Department of Pediatric Infectious Diseases, Malatya, Turkey.

b. Selcuk University Faculty of Medicine, Department of Pediatric Neurology, Konya, Turkey.

c. Selcuk University Faculty of Medicine, Department of Pediatric Infectious Diseases, Konya, Turkey.

d. Selcuk University Faculty of Medicine, Department of Radiology, Konya, Turkey.

E-mail address:

Gülsüm Alkan, M.D.: galkan-85@hotmail.com

Funding: None.

Conflict of interest: None.

Received: 11-18-2018

Accepted: 6-6-2019

\section{INTRODUCTION}

Behçet's disease (BD) is chronic, relapsing, inflammatory disorder, characterized by recurrent oral aphthous ulcers, genital sores and any of several systemic manifestations (ocular, skin and vascular lesions, neurological disease, arthritis, intestinal ulcers). The underlying pathological lesion is an occlusive vasculitis in arterioles and veins. ${ }^{1}$ The disease is more common in Turkey $(80-370.0$ per 100,000$)$ and other countries in the Middle East. BD is a rare in childhood classified as variable vessel vasculitis. ${ }^{2}$ The clinical spectrum is heterogeneous. Oral ulcers and fever of unknown origin are frequent at onset of disease. There are currently no universally recognized pathognomonic tests or biomarkers for $\mathrm{BD}$. The diagnosis of $\mathrm{BD}$ in adults requires two of the clinical findings in addition to recurrent oral apthae; recurrent genital apthae, eye lesions (uveitis or retinal vasculitis), skin lesions (erythema nodosum, pseudo folliculitis, papulopustular lesions, acneiform nodules), and pathergy test positivity concurrently. ${ }^{1,3}$ Children have generally few symptoms, and the time to diagnosis is long. In pediatrics are used 2 diagnostic criteria: one of the International Study Group (ISG), with a specificity of $96 \%$ and sensibility of $91 \%$, and the Mason- Barnes criteria with a specificity of $84 \%$ and a sensibility of $86 \%$. Although these tests were not validated in pediatrics, they are in use. ${ }^{3}$

Recurrent oral ulceration is the most common presenting feature in children. Twenty to forty percent of children with BD have some articular involvement, generally arthralgia in few joints. Pathergy test is not criterion for BD diagnosis in children. If necessary, pathergy test is use in pediatrics as diagnostic criteria; an indirect way of knowing positivity is to have as antecedent the appearance of papules or pustules in areas that have suffered some microtrauma. The positivity of the HLA-B51 allele is associated with susceptibility to the disease. BD is usually more severe in such patients. ${ }^{4,5}$

\section{CASE REPORT}

A 14 years-old female was admitted with complaints of headache, and three-day history 
of diplopia without fever or nausea. The headache was characterized around the neck area for five days. She had a history of recurrent oral ulcers for two years. Other her medical history was unremarkable. She appeared to be alert. Initial vital signs were within normal limits: body temperature $36.6^{\circ} \mathrm{C}$, heart rate, 75 beats / $\mathrm{min}$; blood pressure, $100 / 60 \mathrm{mmHg}$; and respiratory rate, $20 / \mathrm{min}$. Her weight and height were $47 \mathrm{~kg}$ (percentile 10-25) and $152 \mathrm{~cm}$ (percentile 3-10), respectively. At admission physical examination, bilateral papilledema and meningeal irritation was positive, and the result of other system examinations was unremarkable. Brain magnetic resonance imaging (MRI) revealed pachymeningeal enhancement with leptomeningeal contrast-enhancement (Figure 1a).

Lumbar puncture showed high intracranial pressure $\left(640 \mathrm{mmH}_{2} \mathrm{O}\right)$. Cerebrospinal fluid (CSF) analysis revealed 330 cells $/ \mathrm{mm}^{3}$ with lymphocyte, protein and glucose, chloride contents were $161 \mathrm{mg} / \mathrm{dL}, 63 \mathrm{mg} / \mathrm{dL}$ and 129 $\mathrm{mmol} / \mathrm{L}$ respectively. Her primary laboratory data were as follows: white blood cell counts 9,100/ $\mathrm{mm}^{3}$, neutrophils $75 \%$; hemoglobin $15 \mathrm{~g} / \mathrm{dL}$; platelets $396,000 / \mathrm{mm}^{3}$; erythrocyte sedimentation rate, $7 \mathrm{~mm} / \mathrm{h}$ and C-reactive protein $0,1 \mathrm{mg} / \mathrm{dL}$. Serum biochemical analysis was normal. Based on these clinical and laboratory findings, the patient was diagnosed with acute aseptic meningitis. The patient was treated with ceftriaxone and acetazolamide. CSF polymerase chain reaction (PCR) for herpes simplex virus, Streptococcus pneumoniae, Neisseria meningitidis, Haemophilus influenzae, and Enterovirus were all negative. CSF culture was negative. Serum viral serology was unremarkable. With no improvement in clinical findings, lumbar puncture was repeated after one week. The CSF remained increased pressure, and 495 cells $/ \mathrm{mm}^{3}$ with predominance of lymphocyte, $180 \mathrm{mg} / \mathrm{dl}$ protein, $80 \mathrm{mg} / \mathrm{dl}$ glucose. CSF PCR for tuberculosis, acid-fast bacillus stain, and agglutination test and PCR for Brucella were negative. Serology results for Lyme, Rickettsia and Brucella in the blood were negative. The result of tuberculin skin testing was negative. Chest X-ray and abdominal ultrasonography were normal. CSF neuromyelitis optica antibodies and oligoclonal bands were negative. Immunological screening tests were normal. The markers for autoimmune and connective tissue disorders (i.e., antinuclear antibodies, anti-dsDNA, C3, $\mathrm{C} 4$, Coombs, anticardiolipin antibody, thyroidstimulating hormone, and free T4) were all negative. Sarcoidosis was ruled out by the laboratory tests. When the patient's history was further investigated for $\mathrm{BD}$, she remembered to be diagnosed arthritis five years ago. No remarkable disease was recorded in the family history. Ophthalmological examination revealed no uveitis. Pathergy test was negative. MRI venogram was normal. The patient was found to be positive for the HLA-B51 allele. The clinical findings and the laboratory abnormalities were consistent with the diagnosis of non-parenchymal NBD. The patient received high dose intravenous methylprednisolone for 5 days, followed by oral

FIGURE 1. MRI of the brain. (A) Coronal T1 post-gadolinium images shows pachymeningeal enhancement. (B) In 1 months after the steroid therapy, follow-up MRI demonstrated nearly complete regression of the pachymeningeal enhancement

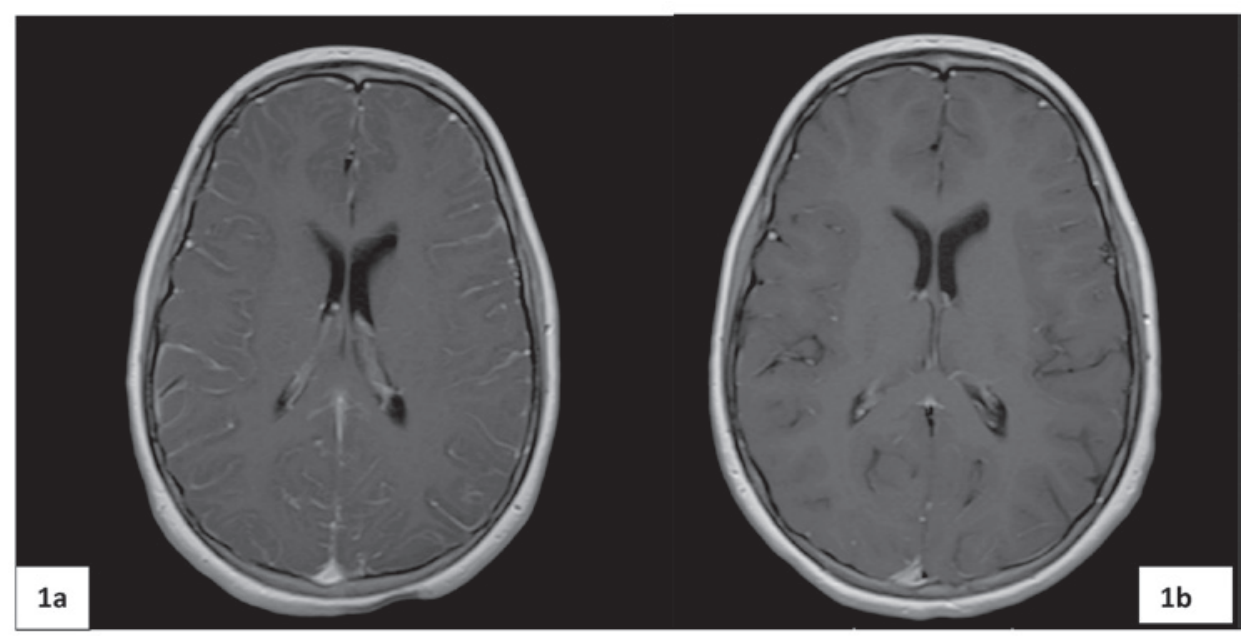


prednisolone (60 mg). On two-day treatment, her headache and diplopia gradually improved. Oneweek later papilledema disappeared completely. A follow-up brain MRI showed nearly complete regression of the leptomeningeal enhancement (Figure $1 b$ ). The CSF parameters improved to 33 cells $/ \mathrm{mm}^{3}$ with predominance of lymphocyte and a protein level of $74 \mathrm{mg} / \mathrm{dL}$, intracranial pressure was normal. Prednisolone was gradually tapered and discontinued one months later. The patient is symptom-free during 6 months on follow-up.

\section{DISCUSSION}

The central nervous system involvement of the disease is known as NBD that presents variable neurological manifestations. Clinic spectrum include dementia, psychiatric symptoms, cranial nerve palsy, cerebellar ataxia and pyramidal tract signs. Parenchymal brain disease is the most common manifestation. The nonparenchymal involvement is usually a benign intracranial hypertension with papilledema, meningitis, or dural sinus thrombosis, with good prognosis. ${ }^{6}$

NBD is uncommonly reported in childhood, ranges from $5 \%$ to $15 \%$. Headache is the most common neurological symptom. Although cerebral venous thrombosis, and cranial nerve palsy are common, meningitis is relatively rare., ${ }^{3,7}$ Our patient had an acute headache and presented a clinical picture characterized by lymphocytic meningitis. The clinical history, biochemical and instrumental exams suggested meningitis. Initially, suspicion of an infectious ethology led to introduce an anti-infectious treatment.

There are no diagnosis criteria or pathognomonic radiological features for $B D$ in children. MRI is the most sensitive imaging technique for diagnosis. In adult patients with NBD, MRI shows lesions mainly in the brainstem, basal ganglia, and white matter. A study on childood parenchymal NBD, MRI findings include brainstem and / or diencephalic lesions were frequently identified whether the lesions are associated with periventricular or subcortical. ${ }^{8-9}$ Pachymeningitis is a rare chronic inflammatory meningeal disorder characterized by a diffuse or localized thickening of the dura mater, caused by various infectious, autoimmune or malignant diseases..$^{10}$ Althought there have been few reports describing pachymeningitis with NBD in adults, there are none described in children.

Diagnosis of NBD is challenging for pediatricians; it may lead to irreversible complications. In our patient, it was extremely difficult to suspect NBD initially. On first investigation, there was no clinical improvement to antibiotic treatment. Autoimmune, connective tissue disorder markers and, neoplastic workups were negative in blood and CSF. Advanced research for meningitis, no infectious agent detected. Her medical history was detailed. Recurrent oral ulcer, aseptic meningitis, and history of arthritis attack were considered to NBD. Ophthalmological examination was normal and pathergy test was negative. Pathergy test is not necessary for $\mathrm{BD}$ diagnosis in children unlike adults. HLA-B51 allele positivity supported to diagnosis. Radiological and clinic recovery was seen rapidly after pulse steroid treatment.

To date, treatment of NBD remains largely empirical. Non-parenchymal with NBD have a more favorable prognosis, those manifestations (such as pachymeningitis) can be managed with steroids alone. Steroids should be combined with an immunosuppressant drug in refractory response. Our patient clinical recovery was quickly to steroid. ${ }^{11}$

NBD, like other systemic disorders (infection, autoimmune, malignancy) may cause pachymeningitis, and can present with aseptic meningitis. BD must always be included in a differential diagnosis of acute meningeal syndrome unless an infectious agent is demonstrated.

In our patient, the diagnosis is by exclusion of more frequent pathologies and by the excellent response to corticosteroid therapy. Early diagnosis and treatment are important for minimize mortality and morbidity.

\section{REFERENCES}

1. International Study Group for Bechet's disease. Criteria for diagnosis of Behcet's disease. Lancet. 1990; 335(8697):1078-80.

2. Koné-Paut I. Behçet's disease in children, an overview. Pediatr Rheumatol Online J. 2016; 14(1):10.

3. Ozen S. Behçet Disease. In Petty R, Laxer R, Lindsley C, Wedderburn L. Textbook of Pediatric Rheumatology. 7th ed. Philadelphia: Saunders. 2016; 40:526-32.

4. Sharma A, DeD, Vaiphei K, Dalai R, Ghosh A. Behçet disease in a child: A rare disorder with an unusual complication and favorable outcome. Indian Dermatol Online J. 2018; 9(2):123-5.

5. Maldini C, Lavalley MP, Cheminant M, de Menthon M, Mahr A. Relationships of HLA-B51 or B5 genotype with Behcet's disease clinical characteristics: systematic review and meta-analyses of observational studies. Rheumatology (Oxford). 2012; 51(5):887-900.

6. Uluduz D, Kürtüncü M, Yapici Z, Seyahi E, et al. Clinical characteristics of pediatric-onset neuro- Behçet's disease. Neurology. 2011; 77(21):1900-5. 
7. Sakakibara R, KoideN, Kishi M, Ogawa E, Shirai K. Aseptic meningitis as the sole manifestation of Behcet's disease. Neurol Sci. 2009; 30(5):405-7.

8. NanthapisalS, Klein NJ, Ambrose N, Eleftheriou D, Brogan PA. Paediatric Behçet's disease: a UK tertiary centre experience. Clin Rheumatol. 2016; 35(10):2509-16.

9. Mora P, Menozzi C, Orsoni JG, Rubino P, et al. NeuroBehçet's disease in childhood: A focus on the neuro- ophthalmological features. Orphanet J Rare Dis. 2013; 8:18.

10. Yoon BN, Kim SJ, Lim MJ, Han JY, et al. Neuro-Behçet's Disease presenting as Hypertrophic Pachymeningitis. Exp Neurobiol. 2015; 24(3):252-5.

11. UralO, GençE, Demir NA, BalcıB, GençBO. Neuro-Behçet's syndrome presenting with features mimicking acute tuberculous meningitis. Int J Infect Dis. 2009; 13(4):e141-4. 